\title{
Bioactive and Tribological Behaviour of Atmospheric Plasma Sprayed Hydroxyapatite Coatings Reinforced by Lanthanum Oxide
}

\section{Yugeswaran Subramanian a,, P.V. Ananthapadmanabhan b, Konstantinos M. Paraskevopoulos c, Akira Kobayashi d, e}

${ }^{a}$ Department of Physics, Pondicherry University, Pondicherry - 605014, India

b Director Research, Sri Shakthi Institute of Engineering and Technology, Coimbatore 641062, Tamil Nadu, India

c Department of Physics, Aristotle University of Thessaloniki, 54124 Thessaloniki, Greece

${ }^{d}$ Department of Physics, Faculty of Science, Chulalongkorn University, Bangkok 10330, Thailand

e JWRI, Osaka University, 11 1 Mihogaoka, Ibaraki, Osaka 567 0047, Japan.

*Corresponding Author yugesh.phy@pondiuni.edu.in (Yugeswaran Subramanian)

Received : 20th April 2019 Accepted : $31^{\text {st }}$ May 2019

ABSTRACT: Lanthanum oxide $\left(\mathrm{La}_{2} \mathrm{O}_{3}\right)$ reinforced Hydroxyapatite coating was deposited by using unique gas tunnel type plasma spray torch under optimum spraying conditions. The phase and microstructure of the as prepared powder and coatings were characterized by $\mathrm{X}$ ray diffraction (XRD) and scanning electron microscope (SEM). In vitro bioactivity of the plasma sprayed lanthanum oxide reinforced hydroxyapatite coatings were investigated by using simulated body fluid solution. Results showed that there was onset of apatite formation on the surface of coatings after 15 days of immersion in SBF, while after 19 days of immersion in SBF it was indicated that a HCAp phase crystallized on their surface. Our studies demonstrate that lanthanum oxide reinforced hydroxyapatite coatings are potentially useful biomaterials with good tribological and bioactive behaviour.

Keywords: Hydroxyapatite, Lanthanum oxide, Plasma spraying, Coating, Bioactivity, SBF, Wear resistance

\section{Introduction}

Hydroxyapatite (HA) is bioactive as it allows for bone cell growth on its surface [1]. HA has close similarities with the inorganic mineral component of bone and teeth and possesses exceptional biocompatibility and unique bioactivity [2]. The use of hydroxyapatite coatings on the metal implants has been widely adopted for use in clinical studies, because the HA coating can achieve firm and direct biological fixation with the surrounding bone tissue [3]. On the other side, HA has poor bending strength and fracture toughness. Due to the latter properties it is most likely to be unsuitable for use in applications that are needed to withstand the applied load, for example, applications occurring at the hip joint. Therefore, HA is applied as a coating on a stronger substrate, usually metallic, which can provide higher strength and fatigue resistance [1]. A number of different methods have been used for the production of hydroxyapatite coatings. Thermal spraying techniques, such as plasma spraying, have been used for HA coating production from past many years. Plasma technology has in recent years emerged as a novel technique for manufacturing of newer and better materials and it is well known that plasma processing has been an efficient technique for the synthesis of new types of materials, making material processing techniques possible [4]. However, the long-term mechanical properties of HA coatings is a major concern for long term clinical application. Due to this, other metallic implants are favoured because of their greater durability in comparison to synthetic HA. Although these implants can withstand higher loads, they may corrode when implanted [5 8]. The concept behind production of these coatings is to combine the bioactivity of HA with the high mechanical properties of the metallic additions. Meantime, decomposition of phases during the thermal spraying causes less bioactive than crystalline HAP. Hence, there is a need to find a suitable HA composite which is more stable than HAP powder at high temperatures as a feedstock powder for thermal spraying. Previous report by Shin Ike et al. [9] confirms that the tensile and bending strengths of HA can be improved via $\mathrm{La}_{2} \mathrm{O}_{3}$ doping to HAP structure.

Furthermore, Fresa et al. studies states that small amounts $\mathrm{La}_{2} \mathrm{O}_{3}$ can be substituted in $\mathrm{HA}$ microstructure without suppressing the bioactivity [10]. Therefore, the tribological and bioactivity of such coatings needs to be further investigated. The aim of the present work is to deposit lanthanum oxide on reinforced Hydroxyapatite coatings and to evaluate their tribological and in vitro bioactivity after immersion in $\mathrm{C} \sim \mathrm{SBF}$. 


\section{Experimental Procedure}

\subsection{Plasma Spraying}

Specially designed gas tunnel type plasma spray torch was employed to form the coatings with controlled microstructure under optimized operating conditions. The typical torch operating parameters are listed in Table1. Graphical image of the gas tunnel type plasma jet and splats formation during spraying is shown in Fig1. Commercially available $\mathrm{HA}$ and $\mathrm{La}_{2} \mathrm{O}_{3}$ powders with an average particle size of 10 45 um and 2 10 $\mu \mathrm{m}$ respectively were used for the purpose. The microstructure of the HA particles were spherical in shape whilst the $\mathrm{La}_{2} \mathrm{O}_{3}$ powders were angular and both the powders have relatively high purity and are well crystallized. The HA powder were mechanically milled together with $10 \mathrm{wt} \%$ of $\mathrm{La}_{2} \mathrm{O}_{3}$ and the mixture was used as the powder precursor for spraying (labelled as HL10 powder). Herein, the powder precursor was fed externally at the exit of the nozzle in order to avoid decomposition process due to melting in high temperature plasma jet. The coating was formed on the surface of a 316 stainless steel substrate whose dimensions were $50 \times 50 \times 2.5 \mathrm{~mm}$. Prior to spraying, the substrate surface was grit blasted with alumina for surface roughening and was followed by cleaning using acetone. Furthermore, the operating parameters used in the present coating were selected in order to get coating with thickness of around $200 \mu \mathrm{m}$ with significant amount of porosity. The coating is labelled as HL10 coating.

\subsection{Phase, Microstructure and Mechanical Characterization}

Phase constituents of the coatings were identified using JEOL JDX 3530M X ray diffractometer with $\mathrm{Cu} \sim \mathrm{K} \alpha$ radiation source at a voltage of $40 \mathrm{kV}$ and a current of 40 $\mathrm{mA}$. The microstructures of the coatings were examined by ERA8800FE scanning electron microscope equipped with energy dispersive $\mathrm{x} \sim$ ray spectroscopy. Porosity of the coatings was evaluated by image analyzing method using computerized optical microscope. Peel off test method was used to determine the adhesive strength of the coatings. The standard specimens with dimensions of $1 \times 1 \mathrm{~cm}$ were used for the study. A ball on disk tribometer was used for estimating the friction and wear resistance of the different coating specimens. All the measurements were carried out at room temperature in laboratory air with a relative humidity of about $60 \%$. Alumina balls, $5 \mathrm{~mm}$ in diameter, were used as the counter body.

Prior to each test the samples and the balls were cleaned with ethanol. The tests were performed using three different normal loads such as 10,20 and $30 \mathrm{~N}$, corresponding to Hertzian stresses within 0.68 1.05 GPa. In all the tests, the sliding speed was maintained at a constant value of $0.2 \mathrm{~ms}^{-1}$ by adjusting the rotation speed of the disk and the diameter of the wear track. Wear volumes of the coating specimens were measured accurately using a three axis profilometer (Taylor Hobson) equipped with a PC. Wear tracks were mapped and the wear volume was calculated accordingly.

Table 1. Typical plasma spraying parameters

\begin{tabular}{|c|c|}
\hline Arc power: & $15 \mathrm{~kW}$ \\
\hline Spraying distance: & $60 \mathrm{~mm}$ \\
\hline Working gas flow rate (Ar): & $1801 / \mathrm{min}$ \\
\hline Carrier gas flow rate (Ar): & $101 / \mathrm{min}$ \\
\hline Powder feed rate: & $15 \sim 20 \mathrm{~g} / \mathrm{min}$ \\
\hline Traverse number: & 16 times \\
\hline
\end{tabular}

The results reported in this paper are in the form of Archard's specific wear rate $\left(\mathrm{mm}^{3} / \mathrm{Nm}\right)$, calculated by the following formula:

Specific wear rate $=\frac{V}{F \times S}$

Where Vis the volume worn away in $\mathrm{mm}^{3}, \mathrm{~F}$ is the normal load in $\mathrm{N}$, and $\mathrm{S}$ is the sliding distance in meter. Each specified test was conducted in three different areas of the coating surface in order to obtain statistically working results.

\subsection{In vitro bioactivity analysis}

The in vitro bioactivity of the coated samples was tested for 5, 10, 15, 19, 25 and 30 days in SBF solution prepared as described in literature [11]. Fourier Transform Infrared Spectroscopy (FTIR) was used to characterize the coatings surface after immersion in the SBF solution for different periods along with XRD. Here, FTIR transmittance spectra were obtained using IFS 113v, Bruker spectrometer in MIR region with a resolution $2 \mathrm{~cm}^{-1}$. The topographical evaluation and elemental analysis of the samples before and after immersion in SBF were performed by a Philips XL40 instrument Scanning Electron Microscope equipped with

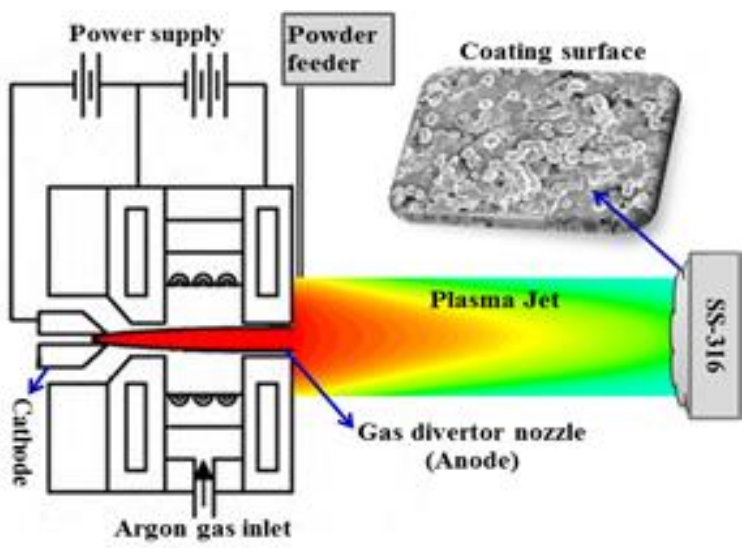

Energy Dispersive X Ray Spectrometer (SEM/EDS).

Fig 1. Schematic of gas tunnel type plasma spray torch 


\section{Results and Discussion}

\subsection{Phase and microstructure formation}

The XRD patterns of the HL1O powder and the as sprayed HL10 coating is shown in Fig2. The XRD spectra showed that the as sprayed pure HL10 coating retained its maximum crystalline HA phase as that of the initial feed stock HA powder, even though there existed a possibility for occurrence of decomposition during its in flight in high temperature plasma zone by the following reaction mechanism [13]:

$$
\begin{gathered}
\mathrm{Ca}_{10}(\mathrm{OH})_{2}\left(\mathrm{PO}_{4}\right)_{6}\{H A\} \longrightarrow 2 \mathrm{Ca}_{3}\left(\mathrm{PO}_{4}\right)_{2}\{T C P\}+ \\
\mathrm{Ca}_{4} \mathrm{P}_{2} \mathrm{O}_{9}\{T T C P\}+\mathrm{H}_{2} \mathrm{O}
\end{gathered}
$$

Conventional plasma sprayed HA coatings generally contain secondary phases such as $\alpha \sim$ TCP, $b \sim$ TCP, TTCP, amorphous calcium phosphate (ACP) and $\mathrm{CaO}$ because of the severe decomposition of the in flight apatite induced by the high temperature plasma jets. On the contrary, in this study the decomposition processes of in flight HA could be well controlled by means of selective operating parameters and the unique nature of the gas tunnel type plasma spray torch. However, the high temperature and heterogeneous nature of plasma jet slightly affected the crystallinity of HA and produced fraction of $\alpha \sim \mathrm{TCP}$ as an additional phase along with $\mathrm{La}_{2} \mathrm{O}_{3}$ phase, which is shown in the XRD pattern of HL10 composite coatings. In general, the decomposition of HA produces $\alpha$ TCP but it's an unstable phase at room temperature and hence obviously gets transformed to $\beta \sim \mathrm{TCP}$, which is a stable phase at room temperature. Here, the presence of small fraction of $\alpha \sim$ TCP phase instead of $\beta \sim$ TCP was due to the rapid solidification behaviour of the gas tunnel type plasma jet that permitted the formation of a stable $\alpha$-TCP. Moreover, in our study the other decomposition phases such as TTCP and $\mathrm{CaO}$ phases were not found. Similar kind of result was previously reported during formation of conventional plasma sprayed HA and its composite coatings [13, 14].

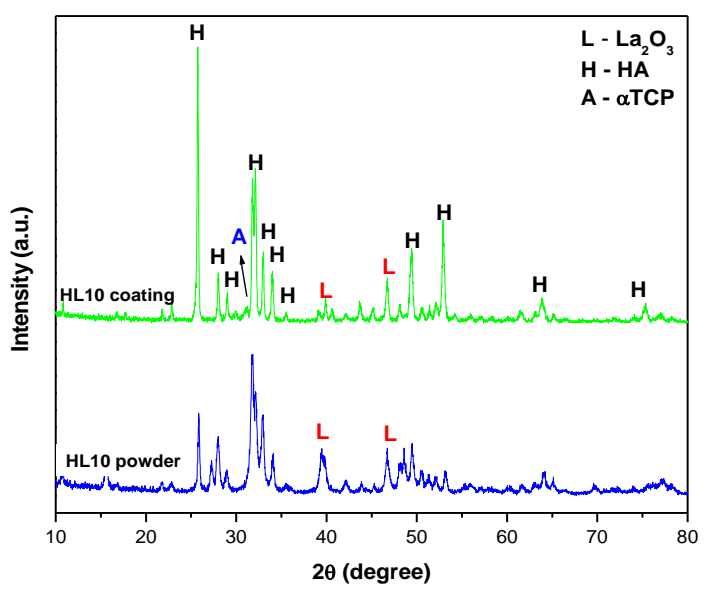

Fig 2. XRD patterns of HL10 powder and gas tunnel type plasma sprayed coating

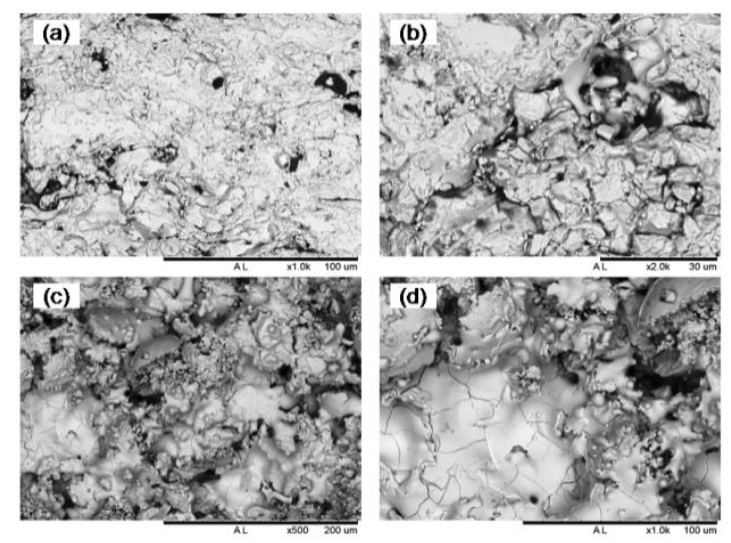

Fig 3. Cross section $(a, b)$ and Surface (c, d) SEM microstructure of HL10 coating

The gas tunnel type plasma sprayed HL10 coating exhibited the presence of characteristic FTIR peaks like that of the synthetic hydroxyapatite (HA) reported in literature [15]. From the spectra, it was found that all the coatings showed a wide peak at $1050 \sim 1100 \mathrm{~cm}^{-1}$ which can be attributed to the stretching vibrational mode of $\mathrm{P} \sim \mathrm{O}$ of $\mathrm{PO}_{4}$ group. In addition, the double peaks at $587 \mathrm{~cm}^{-1}$ and 601 $\mathrm{cm}^{-1}$ attributed to the bending vibrational mode of $\mathrm{P} \sim \mathrm{O}$ bond was observed [16]. Moreover, the HL10 coating revealed a peak at $587 \mathrm{~cm}^{-1}$ and a peak at $603 \mathrm{~cm}^{-1}$ due to the bending vibrational mode of $\mathrm{P} \sim \mathrm{O}$ bond. These findings are in accordance to the FTIR spectra of biological apatite as shown in literature. Although the small amount of $\mathrm{La}_{2} \mathrm{O}_{3}$ in the HL10 coating makes it difficult to distinguish between the peaks attributed to HA and or it's composite respectively. There seems to be a broad peak between $418 \mathrm{~cm}^{-1}$ and 466 $\mathrm{cm}^{-1}$ which can be attributed to the vibrational stretching of $\mathrm{La} \sim \mathrm{O}$ bond [17].

Cross sectional and surface SEM microstructure of as sprayed HL10 coating is shown in Fig 3. It is observed from the micrographs that the coating microstructure is built with typical lamellas and pores with some partially melted and few un melted particles. Plasma sprayed ceramic coatings are generally porous and this characteristic is beneficial to the biomedical application involving the mechanical fixation through bony in growth. Meanwhile, the formation of such pores severely affects the mechanical properties of the coating and as well instigates swift failure. Herein, the investigation results reveal that the porosity and bonding strength of plasma sprayed HL1O coating is around $7 \sim 9 \%$ and $7 \mathrm{MPa}$ respectively. The presence of un melted particles and pores inside the coating microstructure play a vital role in the bonding strength of the coating. Consistent formation of micro cracks inside the coating layer due to the thermal stress and residual coating stresses arising due to the high solidification rate; also the formation of amorphous layer over the surface of the splats and interface during the impinging of in flight droplets to the substrate can reduce the adhesive strength because of the brittle nature. Here, the 
reinforcement of $\mathrm{La}_{2} \mathrm{O}_{3}$ decreases the solidification rate of solution for 5, 10, 15, 19, 25 and 30 days. FTIR spectra the in flight HA particles and prevents the formation of shown in Fig 4 revealed that after 10 days of immersion the amorphous interface layer thereby causing enhancement of onset of apatite formation did not occur since there was no the adhesive strength of the coatings [12].

\subsection{In Vitro Bioactivity}

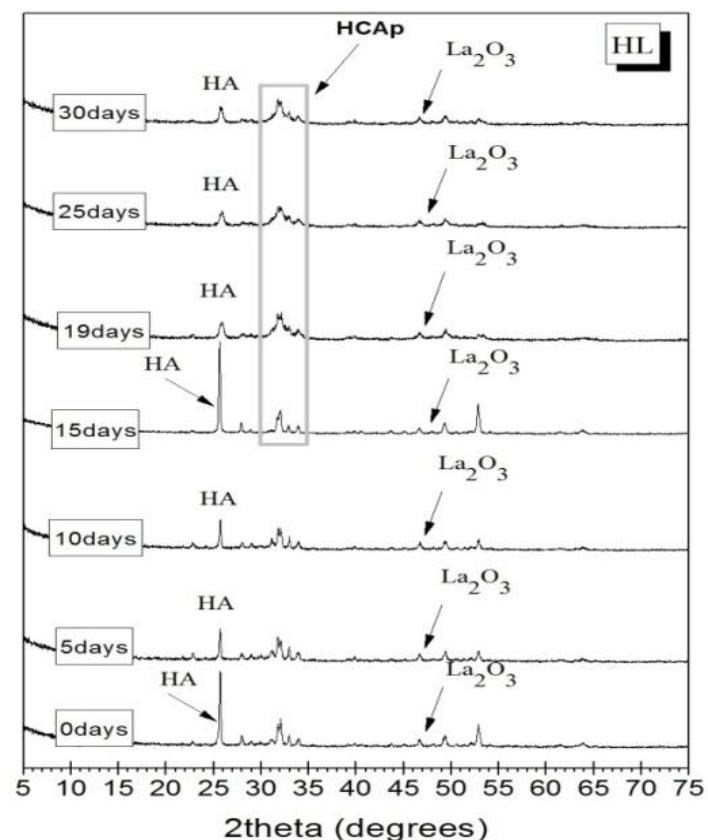

Fig 4. XRD pattern of HL10 coating before and after immersion in SBF for 5, 10, 15, 19, 25 and 30 days.

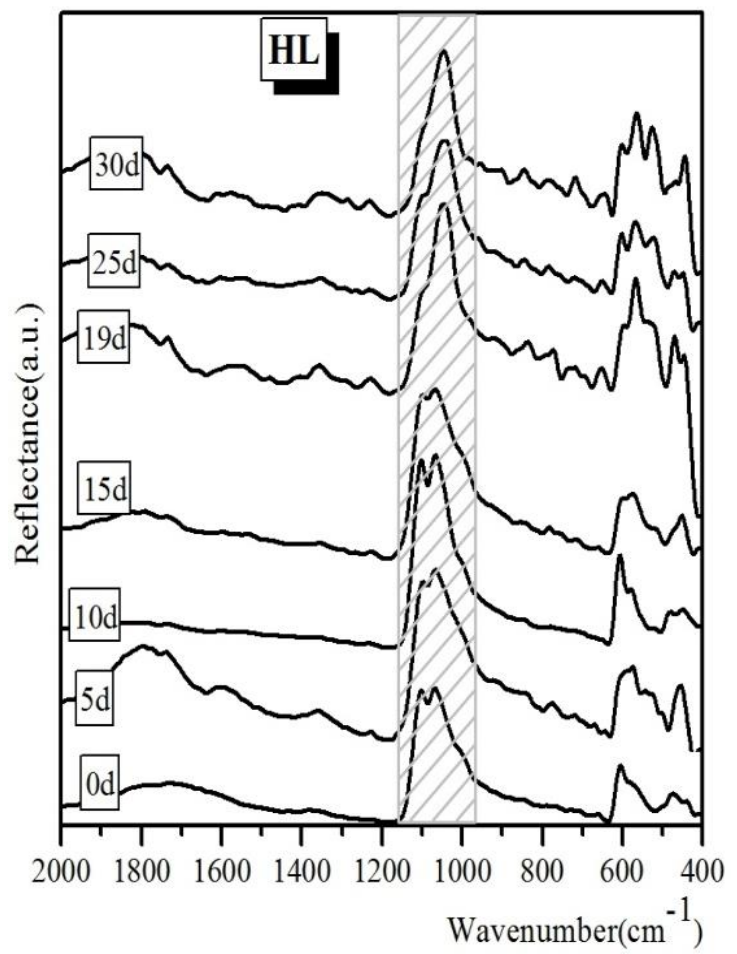

Fig 5. FTIR spectra of HL10 coating before and after immersion in SBF for 5, 10, 15, 19, 25 and 30 days.

Fig 4 and 5 present the XRD patterns and the FTIR spectra of HL10 coatings before and after immersion in SBF significant alteration of the spectra. Moreover after 15 days, the FTIR spectra indicates the beginning of the formation of a Ca $\sim P$ phase associated to the initialization of presence of the double peak at $980 \mathrm{~cm}^{-1}$ and $1150 \mathrm{~cm}^{-1}$, attributed to the stretching vibrational mode of $\mathrm{P} \sim \mathrm{O}$ of $\mathrm{PO}_{4}$ group. After 19 days of immersion in SBF solution, the shifting and the sharpening of the same double peak indicates the onset of apatite formation on the surface of the composite coating. For the same samples, XRD patterns revealed the onset of apatite after 15 days in agreement with the FTIR spectra.
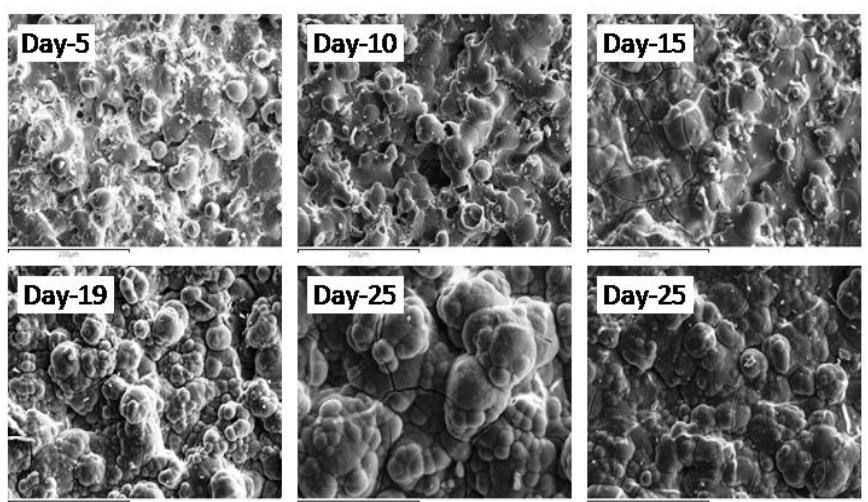

Fig 6 SEM micrographs of HL10 coatings after immersion in SBF for $5,10,15,19,25$ and 30 days.

Fig 6 shows the SEM micrographs of HL10 coating surface after immersion in SBF for 5, 10, 15, 19, 25 and 30 days. SEM microphotographs of HL10 coatings did not reveal any formation of apatite on the surface of the samples after 10 days of immersion. Conversely, it was confirmed that after 15 days a $\mathrm{Ca} \sim \mathrm{P}$ phase was sparsely formed on their surface; moreover, after 19 days a HCAp phase crystallized on their surface. These findings are confirmed by EDS analysis, which revealed a mean molar $\mathrm{Ca} / \mathrm{P}$ ratio of about 1.82 for samples immersed in SBF for 15 days compared to samples immersed for 30 days which revealed a mean molar $\mathrm{Ca} / \mathrm{P}$ ratio of about 1.76 .

\subsection{Sliding wear behaviour}

Sliding wear rate of HL10 coatings at different applied loads $(10,20$ and $30 \mathrm{~N})$ is shown in Fig 6 in comparison with pure HA coating (HLOO) and 30 wt.\%La2O3 reinforced HA coating (HL30). It was observed that the sliding wear rate got considerably reduced in HL1O coating rather than pure HA and HL3O coatings. This decreasing trend can be attributed to the porosity and hardness of the coating microstructure with respect to the $\mathrm{La}_{2} \mathrm{O}_{3}$ weight percentage. In case of HL30 coating, the result revealed maximum wear rate at all the load conditions. As a rule, the sliding wear behavior of the plasma sprayed coatings is largely influenced by the volume fraction of porosity and the pore size distribution. However, the wear 
conditions determine the constructive and or destructive role of porosity in wear resistance. For instance, in dry sliding wear conditions, the porosity can promote surface and subsurface cracking and also favour the occurrence of granular wear particles in the contact area. Here the measured porosity of the plasma sprayed HL30 coating microstructure is around $18 \%$. Meanwhile, it was found that the sliding wear rate of HLOO, HL1O and HL30 coatings significantly increased by increasing the wear load, which can be related to the changes in wear mechanism on the coating surfaces. Herein, on all the coating surfaces instead of abrasive wear mechanism, adhesive and or third body wear mechanism can also occur. At high wear load, the feeble bonding strength of the partially or un melted HA particles causes it to be easily disconnected from its lamella microstructure. Further breaking of the bond junctions occurs due to the continuous motion of the surfaces and creates wear of particles from the weaker materials; this is the stand point of adhesive wear mechanism during sliding at high load conditions. Also, the third body wear mechanism is sometimes induced by the resulting adhesive wear particles [18].

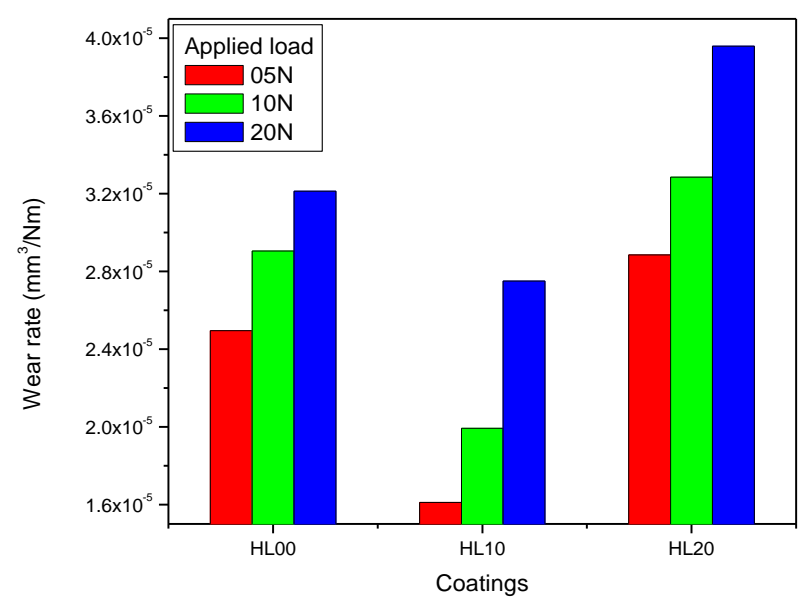

Fig 7. Sliding wear rate of HL10 coating at different applied loads in comparison with HA coating (HLOO) and 30 wt.\%La2O3 reinforced HA coating (HL3O).

The cohesive strength of the splats in the lamella microstructure is significantly enhanced due to the reinforcement of $\mathrm{La}_{2} \mathrm{O}_{3}$ which prevents the removal of HA splats from the coating microstructure. Fig 6 shows the 3D image of wear track surface of HL1O and HA coatings at 10 and $30 \mathrm{~N}$ wear load conditions. It is seen that, at low wear load, the subsurface deformation is negligible due to the type of wear mechanism and grooves formation, but at high wear load, the depth of the grooves formed from the abrasive particles of the coating surface is high and thus encourages deformation over the surface. Furthermore, the change in wear mechanism with respect to the applied wear loads is clearly evident from the wear track surface of the coatings at two different wear load conditions $[19,20]$.

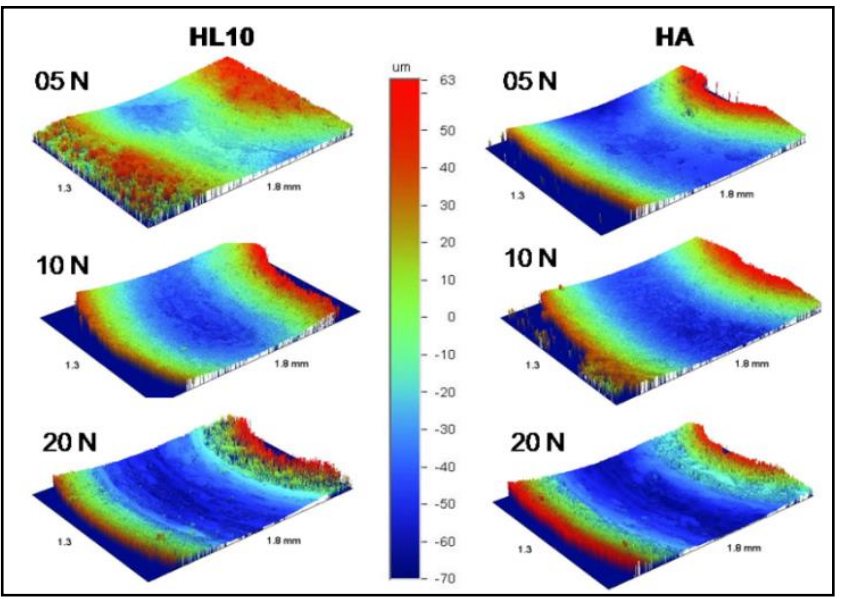

Fig 8. 3D image of wear track surface of HL10

coating at different applied loads in comparison with HA coating (HLOO).

\section{Conclusion}

Crystalline hydroxyapatite coating with $10 \mathrm{wt} . \%$ lanthanum oxide reinforced were deposited by using unique gas tunnel type plasma spray torch under optimum spraying conditions. The phase and microstructure formation and sliding wear behaviour of the as prepared coating were characterized. In vitro bioactivity of the coating was tested in conventional simulated body fluid (c $\sim \mathrm{SBF}$ ) solution. On the surface of HL10 coatings, after 15 days of immersion in SBF, was revealed the onset of apatite formation, while after 19 days of immersion in SBF it was indicated that an HCAp phase crystallized on their surface. The obtained investigation results showed that the reinforcement of 10 wt.\% $\mathrm{La}_{2} \mathrm{O}_{3}$ in HA significantly enhanced the sliding wear resistance without effecting the bioactivity of the HA surface. Hence, the above composition coating, qualifies as the most suitable biomaterial for bio surface engineering areas.

\section{Reference}

[1] T.J. Levingstone, Optimisation of Plasma Sprayed Hydroxyapatite Coatings, (2008) PhD Thesis, Dublin City University

[2] A.K. Nayak, Hydroxyapatite Synthesis Methodologies: An Overview, Int. J. chemtech res., 2 (2010) 903 907.

[3] T. M. Lee, C.Y. Yang, E. Chang and R.S. Tsai, Comparison of plasma sprayed hydroxyapatite coatings and zirconia reinforced hydroxyapatite composite coatings: in vivo study, $J$ Biomed Mater Res A; 71 (2004) 652.

[4] Hui Yang, Lin Zhang and Ke Wei Xu, The microstructure and specific properties of $\mathrm{La} / \mathrm{HAP}$ 
composite powder and its coating, Appl Surf Sci., 254 (2007) 425-430.

[5] I. Mayer, J.D. Layani, A. Givan, M. Gaft and P. Blanc, La ions in precipitated hydroxyapatites, J. Inorg. Chem., 73 (1999) 221-226.

[6] F. Fernandez Gavarron, T. Huque, J.L. Rabinowitz and J.G. Brand, Incorporation of 140 lanthanum into bonesa, teeth and hydroxyaptite, Bone Miner., 4 (1988) 283-291.

[7] R. McPherson, N. Gane and T.J. Bastow, Structural characterization of plasma sprayed hydroxylapatite coatings, J. Mater. Sci.: Mater. Med., 6 (1995) 327-334.

[8] V. Deram, C. Minichiello, R.N. Vannier, A. Le Maguer, L. Pawlowski and D. Murano, Microstructural characterizations of plasma sprayed Hydroxyapatite coatings, Surf. Coat. Technol. 166 (2003) 153-159.

[9] A. Tanaka, Y. Nishimura, T. Sakaki, A. Fujita and T. Shin ike, Histologic evaluation of tissue response to sintered lanthanum containing hydroxyapatites subcutaneously implanted in rats, J Osaka Dent Univ., 23(2) (1989) 111 20.

[10] Weiwei Lou, Yiwen Dong, Hualin Zhang, Yifan Jin, Xiaohui $\mathrm{Hu}$, Jianfeng $\mathrm{Ma}$, Jinsong Liu and Gang $\mathrm{Wu}$, Preparation and Characterization of Lanthanum Incorporated Hydroxyapatite Coatings on Titanium Substrates, Int J Mol Sci., 16(9) (2015) 21070-21086.

[11]T. Kokubo, H. Kushitani, S. Sakka, T. Kitsugi and T. Yamamuro, Solutions able to reproduce in vivo surface structure changes in bioactive glass ceramic A W, J. Biomed. Mater. Res., 24 (1990) 721 34.

[12]S. Yugeswaran, C.P. Yoganand, A. Kobayashi, K.M. Paraskevopoulos, B. Subramanian, Mechanical properties, electrochemical corrosion and in vitro bioactivity of yttria stabilized zirconia reinforced hydroxyapatite coatings prepared by gas tunnel type plasma spraying, J. Mech. Behav. Bio Mater., 9 (2012) 22 33.

[13] B. Lorcardi, U.E. Pazzaglia, C. Gabbi, B. Profilo, Thermal behavior of hydroxyapatite intended for medical applications, Biomaterials 14 (1993) 437 441.

[14]E. Chang, W.J. Chang, B.C. Wang, C.Y. Yang, Plasma spraying of zirconia reinforced hydroxyapatite composite coating on titanium, J.Mater. Sci., 8 (1997) 193 200.

[15]J.M.E. Matos, F.M. Anjos Jïnior, L.S. Cavalcante, V. Santos, S.H. Leal, L.S. Santos Jïnior, M.R.M.C. Santos, E. Longo: Reflux synthesis and hydrothermal processing of ZrO2 nanopowders at low temperature, J. Mater. Chem. Phy., 117 (2009) 455-459.
[16] S. Damyanova, B. Pawelec, K. Arishtirova, M.V. Martinez Huerta, J.L.G. Fierro, Study of the surface and redox properties of ceria zirconia oxides, Appl. Catal., A. 337(2008), p86 98.

[17]A.Aronne, S. Esposito, P. Pernice. FTIR and DTA study of lanthanum aluminosilicate glasses, Mater. Chem. Phys. 51 (1997), 163 168.

[18] Yugeswaran S., Kobayashi A., HikmetUcisik A., Subramanian B., 2015. Characterization of gas tunnel type plasma sprayed hydroxyapatite-nanostructure titania composite coatings, AppI Surf Sci., 347, pp. 48 56.

[19]M. Gaona, R.S.Lima and B.R. Marple, Nanostructured titania / hydroxyapatite composite coatings deposited by high velocity oxy fuel (HVOF) spraying, Mate. Sci. Eng., A 458 (2007) 141 149.

[20]E. Chang, W.J. Chang, B.C.Wang, C.Y. Yang, Plasma spraying of zirconia reinforced hydroxyapatite composite coating on titanium, J. Mater. Sci., 8 (1997) 193 200.

\section{About The License}

(C) 2019 The Authors. This work is licensed under a Creative Commons Attribution 4.0 International License which permits unrestricted use, provided the original author and source are credited. 